\title{
ALGUNOS CAMBIOS DE ORIENTACION METODOLOGICÃ: DESDE Y PARA LA ACCION COLECTIVA Y LA CIUDADANIA*
}

\author{
Tomás R. Villasante $* *$
}

\begin{abstract}
SÍNTESE - O autor propõe uma discussão sobre novos enfoques relativos a movimentos sociais decorrentes das mudanças de paradigmas que atingem, neste final de século, toda ciência. Disso resultam novas propostas e modelos de ação coletiva que possibilitam uma democracia participativa.
\end{abstract}

PALAVRAS-CHAVE - movimentos sociais, cidadania, sociedade civil.
ABSTRACT - The author proposes a discussion about a new focus with respect to social movements that result from the changes of paradigms that affect all sciences at this time, the end of the century. From this come new proposals and models of collective action which make a participatory democracy possible.

KEY WORDS - social movements, citizenship, civil society.

Se trata de dejar sentados algunos nuevos enfoques sobre movimientos sociales que se producen con los cambios de paradigmas que se están pianteando en las ciencias sociales en este fin de siglo. El cambio de paradigmas afecta a todas las ciencias en general y también a las sociales. Cambia la comprensión de la vida cotidiana, desde una crítica a las utopías racionalistas de la modernidad, y por lo mismo está en relación también con los cambios en los propios movimientos sociales. Esto no quiere decir que la crítica sea necesariamente posmoderna, o irracionalista, o esotérica. Hay una serie de nuevas aportaciones desde distintas tradiciones emancipatorias (ecologia, feminismo, filosofia de la praxis, etc.) que apuntan a pensamientos más complejos y más constructivistas socialmente.

No se trata tanto de un nuevo enfoque desde los estudios de los movimientos sociales, como de una crítica a los estudios habituales hecha desde los propios movimientos, ya que muchos de estos estudios son puramente académicos y su servicio a los movimientos los hacen poco operativos, muy rígidos, sobre todo clasificatorios o taxonómicos. Esto esta relacionado por tanto con el paso de los

* Una primera versión de este texto fue enviado para su discusión como ponencia a los Seminarios Internacionales de Vitoria (España) y de Braga (Portugal).

** Director del Curso de Posgrado "Investigación Participativa y Gestión Local". Facultad de Sociologia, Campus de Somosaguas, Universidad Complutense, Madrid.

\begin{tabular}{|l|l|l|l|l|l|}
\hline VERITAS & Porto Alegre & v. 43 & $\mathrm{n}^{0}$ especial & Dezembro 1998 & p. $217-228$ \\
\hline
\end{tabular}


prepuestos objetivistas de las ciencias a los constructivistas y reflexivos, de los presupuestos de esencias simplificadoras de los fenómenos a los de la complejidad de lo social, y de la causa-efecto a las retroalimentaciones entre los investigadores y los actores sociales con sus propias reflexividades.

Aqui vienen a coincidir tanto las necesidades de autocrítica interna de los movimientos como los replanteamientos epistemológicos de las ciencias sociales. No se trata por lo mismo de una crítica superficial a algún aspecto concreto de los enfoques habituales, sino de un replanteamiento de fondo. Esto debería plantearse con un texto mucho más largo y documentado, pero en esta ocasión solo podemos hacer referencia a algunos textos donde se recogen buena parte de las tesis que aquí apuntamos, recopilando escritos e investigaciones anteriores. Nos centramos en enunciar lo que nos parece un replanteamiento necesario dados los retos que se nos presentan, la formulación de las principales críticas y preguntas, y la enunciación de las metodologías que nos parecen más operativas. Si se escribe en plural no es por un tono mayestático, sino por el intento de reconocer que se trata de un trabajo colectivo y en proceso.

\section{De las redes comunitarias y de los movimientos a los conjuntos de acción y conductas paradójicas}

No basta quedarnos en los estudios de redes comunitarias y de movimientos que tanto han abundado en los últimos años. Está bien que haya habido tantos estudios de casos y que se haya profundizado en sus características concretas, sobre todo por lo que ha supuesto de crítica a algunos dogmatismos anteriores, y por la muestra de la complejidad interna de relaciones que se produce en cada movimiento. Pero con toda su importancia estos estudios no han sido casi nunca comparativos, con lo que las posiblidades de pasar de la crítica a aportaciones más generalizables y operativas se queda muy limitada. Precisamente en estos momentos lo que precisariamos es algunas pistas metodológicas con las que abordar comparaciones entre distintas situaciones a veces muy dispares.

La mayor parte de estos estudios además nos proporcionan solo lo que ya hay en cada movimiento o comunidad, lo establecido, dando por bueno causas y efectos hasta el momento presente. Esta forma de acercarse a una supuesta objetividad no tiene en cuenta las reflexividades de los procesos en marcha, incluido el de la propia investigación. La realidad no es algo preexistente que se pueda objetivar y archivar con datos unitarios e incontestables, sino construcciones siempre en proceso, incluso las lecturas que podamos hacer de realidades históricas.

Así nos interesa saber que hay debajo de datos cuantitativos y detrás de expresiones cualitativas, que nos deben permitir lecturas no solo unitarias sino también paradójicas. Es decir, que según como se quieran interpretar pueden servir para diversos sentidos de los movimientos. Aunque unos dirigentes sociales 0 unas crónicas periodísticas nos parezcan indicar unos sentidos, habrá que ver cómo las diferentes redes informales de cotidianeidad nos dan otros posibles sentidos de los mismos hechos. Incluso en las conversaciones con las mismas personas aparecen paradojas, interpretaciones aparentemente contradictorias, cuando estas se están refiriendo a contextos de redes distintas. Por ejemplo, tenemos la 
experiencia de que un mismo grupo pida más policía en su barrio, y al tiempo culpe a la policía de buena parte de sus desgracias. Más que sancionar que se trata de una incoherencia habrá que investigar cuales son los trasfondos en los que se dice.

Esto nos podría llevar a un fuerte relativismo interpretativo como en muchos de los estudios de casos que se han venido haciendo. Pero el hecho de que no se puedan objetivar unos datos como uniformes, con un sentido único, no quiere decir que todo vale. Hay otras formas de validar las investigaciones, por ejemplo, si se consigue que sirvan para construir resultados sociales con los movimientos que los usan. Para eso han de hacerse unas interpretaciones y unas programaciones a partir de los síntomas detectados, y de los socio-análisis planteados. Y aún así no se deducirá que sea la verdad completa, sino que algo es cierto para esa situación, y que en comparación con otras experiencias puede aportar nuevas formas de usar las paradojas.

Lo que tratamos es de huir del relativismo porque nos vuelve poco operativos, aunque se ha de reconocer que tiene razón al criticar los dogmatismos que dividían los movimientos sociales en buenos y malos. Y aún hoy algunos autores dan una definición de los movimientos tan exclusiva que dejan fuera a todos los que no les gustan. Y otros hablan de anti-movimientos, colocando en ellos los que representa valores contrarios a lo que se supone que es la ciudadania de bien. Cada cual puede tener su propio criterio externo a los movimientos para definirlos en un sentido o en otro, según sus contenidos. Pero estos criterios hacen a los movimientos dependientes de tales lógicas, y no autónomos como elementos sintomáticos de la sociedad, y como constructores desde sus complejidades de nuevas formas de entender la vida.

Preferimos hablar del concepto conjuntos de acción antes que de movimientos sociales, porque así no tenemos que entrar en una definición maniquea que nos obligue a dividir los que nos gustan con los que rechazamos, y sobre todo las situaciones intermedias y cambiantes que son las más frecuentes. Los conjuntos de acción nos muestran las relaciones internas entre las diversas conductas de la vida cotidiana, las redes informales y formales, y cómo interactúan con los poderes establecidos. No sitúan una muralla entre unas relaciones sociales y otras, sino que facilitan entender como se pasa de un populismo a un ciudadanismo, o de un gestionismo a una situación de aislamiento. Al menos ese es el intento, al margen de los contenidos explícitos por los que se los pretende definir y clasificar.

Los conjuntos de acción aportan unos mapas de relaciones entre los principales actores en presencia, tanto formales como informales, y cuales son las posibles variantes de sus estrategias particulares. Con lo cual se delimitan unos campos de potencialidades, no fijos, que permiten tanto análisis autocríticos de los propios movimientos como diseños alternativos para futuras estrategias y programaciones. Los mapas de relaciones permiten que investigadores, políticos, dirigentes o simples colaboradores de base, puedan entrar en el diagnóstico y en las aportaciones de mejora tanto para el movimiento como para las situaciones creadas. Cada relación establecida no solo es bi-univoca entre elementos que tienen sus propias paradojas, sino que también es tendencial, y hay que interpretarla en los conjuntos y campos de acción complejos en que se mueve. 
No estamos pues tratando de encontrar lo que está instituido en el estado o la sociedad, sino lo instituyente, lo que se está construyendo en procesos complejos. Encontrar cuales son las relaciones principales que dinamizan las tendencias internas y externas a los movimientos. No es tanto trabajar con otros datos sino ver en los mismos datos, cualitativos y cuantitativos, las otras dimensiones de su complejidad. El construccionismo social tiene aquí uno de sus campos más claros de operatividad. El concepto de praxis en su sentido más reflexivo, que algunos movimientos sociales han usado en la historia con tanto acierto vuelve a tomar aquí toda su profundidad.

Las propias conductas de los actores no pueden ser entendidas en su complejidad desde una lógica lineal dicotómica (de Aristóteles a Hegel) que solo puede reconocer el principio de identidad y no contradicción, o la síntesis de tesis y antítesis, y no las paradojas de que algo pueda ser lo que es y su contrario al tiempo, con varias posibles salidas. Por ejemplo, si introducimos los cuadrados de Kleim o Greimas, de la lingüística, en los discursos de los movimientos veremos que es posible hablar de cuatro conductas posibles, aparentemente paradójicas, pero muy frecuentes en nuestros estudios empíricos, y no solo un eje entre dos polos o síntesis de causas preestablecidas. Las dialécticas práxicas nos abren así grandes posibilidades para un construccionismo social que abra nuevas perspectivas sin abandonar un cierto rigor metodológico.

Por ejemplo no solo se puede ser "converso" (continuista) o "perverso" (reformista), o sea, decir si o no a las demandas del sistema; también se puede decir "ni si, ni no", no aceptar la autoridad de la pregunta e inaugurar otra forma de preguntas, ser "subversivo" al sistema (revolucionario). Pero hay más, lo más frecuente en los movimientos sociales es lo "reversivo", lo rebelde que desborda las lógicas establecidas desde ellas mismas, afirmar y negar al tiempo una identidad/reivindicación, "si pero no". Por ejemplo el derecho a la vivienda se exige sabiendo que el sistema la tiene entre sus declaraciones formales, pero que en su práctica lo que predomina es la especulación, y entonces se desborda con los hechos al sistema ocupando terrenos o edificios, en unas conductas que afirman y niegan a la vez los contenidos del sistema y de los movimientos, mostrando sus paradojas y contradicciones de fondo.

\section{De la sociedad civi/tercer sector al tercer sistema}

El concepto de sociedad civil que Gramsci y otros popularizaron entre la izquierda ha sido recuperado en gran parte por la nueva derecha. Hoy frente al Estado del Bienestar nos encontramos que se plantea un modelo inconcreto de Sociedad del Bienestar que no acaba de saberse si es neoliberalismo o algún libertarismo. Se hace necesario precisar algo este campo de conceptos para poder entendernos. Tercer sector, como concepto, al menos se distingue tanto de lo que es el Estado como de la lógica de Mercado, que son los otros dos sectores con los que se delimita. Es decir, está compuesto por Organizaciones no gubernamentales y no lucrativas, cuando menos según sus estatutos constitutivos. Mientras que con la sociedad civil algunos quieren hacer un frente de todos los intereses contra el Estado, en su versión más neoliberal. 
El tercer sector entonces nos proporciona una lista de asociaciones y otras formas sociales cuya taxonomía, y sus programas y reivindicaciones, nos lo sitúa como un tercer polo para compensar los desajustes de la sociedad, al menos en teoría. Así nos van a aparecer organizaciones por tal o cual contenido explícito (vivienda, feminismo, ecologia, educación etc.) y que según sus características podremos subdividir (clase social de sus miembros, estructura de oportunidad politica, identidades culturales locales, etc.). La lógica causa-efecto no ve que además del contenido explícito hay otros contenidos latentes que también interactúan en estos procesos.

Un movimiento puede estar consiguiendo un éxito en vivienda, por ejemplo, para un determinado grupo social desfavorecido, pero al tiempo puede estar aislándose dentro de la ciudad, o respecto a otras clases sociales, o desmovilizándose en su conciencia social por la forma en que se ha conseguido. Ninguno de estos elementos es una causa o efecto simple, sin que tienen retroalimentaciones complejas. El campo en que se mueven las organizaciones del tercer sector tiene abundantes contradicciones, paradojas e inconcreciones. Es un campo amplio y que parece cobrar cada vez más importancia, pero necesita entenderse mejor tanto en sus relaciones internas como externas.

Hacia fuera el tercer sector se ha querido relacionar con los otros dos sectores, Estado y Mercado, como si estos fuesen claros en sus propias delimitaciones, y como si guardasen líneas de diferenciación nítidas con este tercer sector. Pero con el fenómeno de la globalización, precisamente, no es posible hablar de una coherencia del Mercado, sino de fuertes enfrentamientos entre el mercado financiero y los productivos, entre los globales y los locales, etc. Y el Estado por un lado se desmantela dejando lo social al tercer sector o a municipios endeudados, y por otro se refuerza en acuerdos económicos o militares internacionales. En realidad se refuerza un Estado+Mercado global, una lógica que se contrapone tanto a pequeños estados o municipios grandes como a producciones locales, que deja a que sean asumidos muchos de los problemas sociales locales por el tercer sector.

Estos antagonismos económicos, políticos y culturales hacen que el tercer sector tenga como complementarios a algunos poderes y productores locales, pero siempre que se entienda que hay contradicciones muy fuertes con los procesos dominantes de la globalización. Es decir que no se trata de un mundo ideal que va a dejar que actúe este tercer sector como la nueva panacea salvadora de la humanidad frente a los desastres ecológicos, demográficos, de desigualdades, etc. sino que trata de usar estos espacios como paliativos para que se escondan estos problemas. Es pues un campo muy importante de actuación pero lleno a propósito de problemas de sentido y contrasentidos en pugna. Los Foros mundiales que desde Río de Janeiro hasta Estambul han venido reuniendo a este tipo de organizaciones son una clara muestra de estos retos que tratamos de mostrar.

El concepto de tercer sistema, en cambio, plantea una dimensión un poco más precisa en cuanto que se propone construir otro sistema de valores civilizatorios que no son los dominantes ni en las lógicas de mercado actuales ni en de los estados de tipo redistribuidor realmente existentes. Por ejemplo prioriza la educación/hegemonía de un bloque alternativo de valores y de sujetos sociales más allá 
de los contenidos inmediatos de las organizaciones y movimientos. Se puede pertenecer al tercer sector pero no por eso estar construyendo un tercer sistema, pues esto solo algunos movimientos y en determinadas circunstancias están contribuyendo a ello, cuando coinciden en desarrollar esos nuevos valores antagónicos con los dominantes.

El tercer sistema, para no quedarse aislado, busca entonces redes donde desarrollarse dentro del tercer sector; también con determinadas instituciones de ámbito público, como Ayuntamientos, Universidades, etc. que se puedan ver fuera de los procesos de la globalización militar/económica; e incluso también en los ámbitos del mercado y la producción que tienen contradicciones con la acumulación capitalista trasnacional. Es decir, en zonas no centrales del sistema es posible que funcionen economías de tipo popular apoyadas en programas de desarrollo con ciertas caracteristicas de lo alternativo. No es que esta via pueda hacerse sin conflictos, sino que a muchas realidades periféricas no les queda otra lógica que agarrarse a este tipo de fórmulas ante la falta de soluciones del propio sistema mundial, y que por otra parte son experimentaciones contradictorias de las que se puede aprender muchos elementos alternativos.

De hecho en todos los imperios que en el mundo han sido siempre ha habido movimientos sociales que, localmente, han anticipado lo que acabaría siendo los valores de la nueva cultura civilizatoria. Ante la tecno-estructura actual de los acuerdos de grandes estados+mercados globales también hay grietas por donde se cuelan experiencias de movimientos o de acuerdos de redes del tercer sistema donde se van planteando y construyendo otros valores y otros comportamientos sociales.
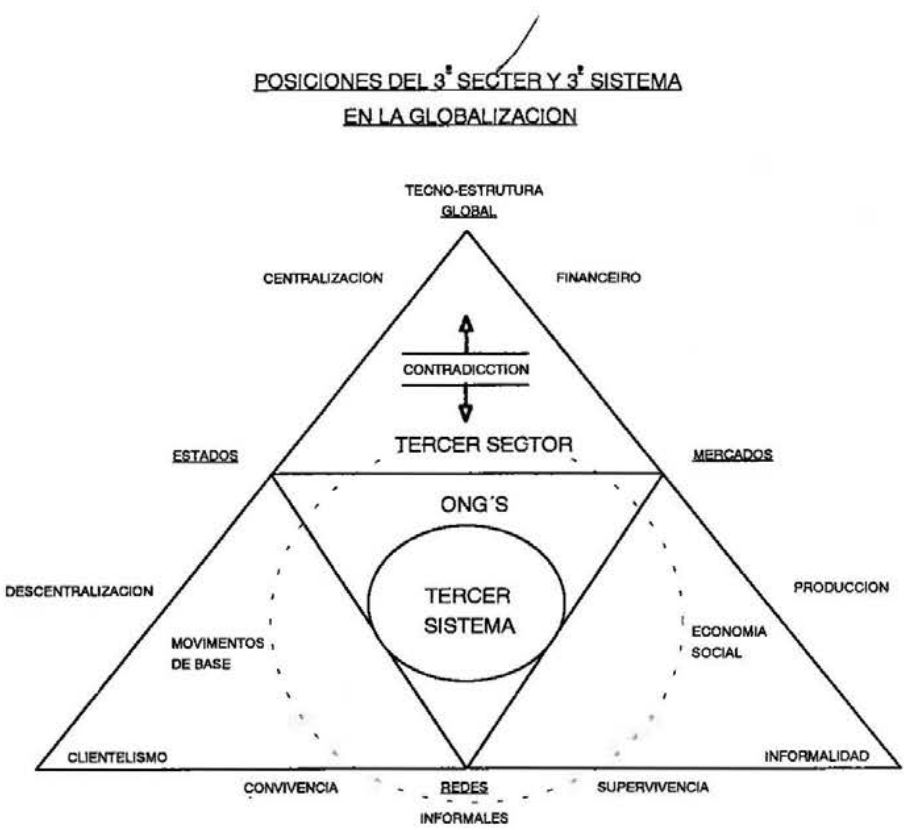


\section{De la participación ciudadana a la democracia participativa}

Entre los valores que se van ensayando entre los movimientos están los referidos a las formas democráticas de funcionamiento, tanto de las propias organizaciones como de la sociedad por la que se quiere transitar. También en esto se han hecho diversas propuestas de participación que en general han cristalizado en reglamentos jurídicos que han venido reconociendo algunos municipios y algunos estados. No es que se haya avanzado mucho pero aún asi se ha llegado a la conclusión de que esta vía poco más da de si. El problema es que la gente no parece querer participar aún con reglamentos muy avanzados, redactados incluso por las propias organizaciones populares. Este hecho viene a plantear nuevas preguntas sobre algunos de los supuestos en que se basaban muchos movimientos sociales y sus reivindicaciones.

Muchas de estas reglamentaciones plantean derechos de consulta a las asociaciones, subvenciones a quienes se inscriben en un listado público, y en general institucionalización de los movimientos. Estos sin duda necesitan ser reconocidos como interlocutores, necesitan apoyos económicos, aspiran a asesorar o incluso a tomar decisiones públicas, etc. Pero cuando lo consiguen a menudo se encastillan en tales posiciones, y hay que esperar que surjan otros nuevos movimientos que dinamicen la vida social. Esto forma parte de las paradojas de los movimientos de las que hablábamos, y solo en su continua dinámica es posible entender sus aparentes contradicciones.

De la empiria sale que cuanto más movilización social hay surgen nuevos dirigentes y hasta nuevos grupos, y que estos tienden a reemplazar a los antiguos, desbordando las organizaciones más constituidas, pero también que cualquier movilización para durar y alcanzar sus objetivos necesita ciertos grados de organización, reconocimiento e institucionalización. Es decir las dos cosas son necesarias aunque tiendan a contraponerse en la práctica, y de ahí las dificultades intrínsecas de todo movimiento que ha de ser al tiempo movilización y organización. Pero justamente de estas paradojas de la complejidad social es de lo que venimos hablando, de las insuficiencias de los análisis clásicos, y de nuevas metodologías para abordarlos.

Mirando la participación ciudadana desde las instituciones habrá que acordar que a penas cuentan con apoyo económico, con técnicas adecuadas, y que el respaldo legal es más consultivo que con real toma de decisiones. Por lo mismo la mayor parte de los ciudadanos, y no pocas organizaciones, no acuden a las convocatorias de participación más que la primera o la segunda vez. No es difícil darse cuenta que en ellas no se encuentra un poder de verdad para hacer cosas. Y si lo ponemos en comparación con los estímulos de la vida cotidiana (medios de comunicación, diversiones etc.) es mejor que, puestos a jugar, sea en algo divertido. Mucha gente sencilla tiene más olfato para estas cosas que muchas organizaciones, y se malician que cuando algunas participan sin resolver temas importantes es porque buscan otros beneficios.

Por eso la construcción de democracias participativas es otra cosa que poco tiene que ver con reglamentos o concejalias de participación ciudadana. Frente a las formas instituidas y legisladas, hay formas democráticas instituyentes, es decir 
procesos en marcha, praxis reflexivas de los agentes sociales, motores para que las iniciativas de grupos de base tomen cuerpo y se desarrollen. Evidentemente no son muy numerosas este tipo de experiencias porque se han de juntar muchas condiciones al tiempo para que puedan funcionar, pero de vez en cuando vemos que hay algunas muestras que demuestran su posibilidad. Por eso no desesperamos de encontrarnos cada vez con mayor precisión algunos de los elementos imprescindibles de tales procesos de democracias instituyentes, aunque solo sea en embrión.

Hay casos de analizadores históricos que funcionan como referentes en cada comunidad, incluso mitificados como ocurre con el mayo del 68 en Francia, o con los movimientos de la transición politica española, o con algunos latinoamericanos. Se pueden y deben analizar estos movimientos pero no para mitificarlos sino para aprender algunas de sus claves internas y externas. Pero cuando las circunstancias externas parece que están en contra de que se puedan repetir tales acontecimientos, hay que centrarse en algunos mecanismos constructivos, algunos ritos o analizadores construidos que puedan provocar pequeños acontecimientos capaces de crear algunas sinérgias sociales.

Es decir para aumentar las probabilidades de que se puedan dinamizar movimientos democrático participativos, se han de juntar una serie de circunstancias mínimas, y aún con ellas no esta garantizado más que un cálculo de las probabilidades en juego. Es decir, entendemos que es posible hacer algunas investigaciones concretas, cualitativas y cuantitativas, que al servicio de lo participativo puedan abrir mejores perspectivas a los procesos instituyentes parciales de cada caso. Por ejemplo algunas investigaciones participantes, socioanálisis, o como nosotros preferimos llamarlas "socio-praxis", nos ilustran de que es posible convertir los Planes comunitarios, programaciones temáticas, campañas sociales, etc. en procesos más allá de la participación ciudadana, intentando al menos que pasen a ser instituyentes desde sus concreciones.

Una primera medida es poner de acuerdo a las partes que tienen voluntad para ello (políticos, expertos, dirigentes, etc.). Y a continuación hay que hacer un mapa de relaciones, no solo de intereses objetivables, sino de habitus y formas convivenciales en redes comunicativas. De ahi los conjuntos de acción de los que hemos hablado nos permiten abrir las estrategias posibles, donde no solo juegan los expertos y dirigentes sino sobre todo la sensibilidad de los sectores populares implicados, con sus rivalidades y aspiraciones incluidas. Con todo ello es posible acordar una estrategia sinérgica para una mayoría de grupos y sectores sociales, y donde lo cultural, territorial, económico, etc. vienen también a reforzar lo programado.

Este tipo de programaciones integrales son las que estamos experimentando y discutiendo participadamente en varios municipios del entorno madrileño, de la misma forma que otros equipos lo vienen haciendo con otro tipo de planes de participación en otros puntos de todo el mundo. No hay seguridad de que se vayan a producir los resultados sinérgicos programados más que en una parte, pero de lo que estamos seguros es que por lo menos ponemos unas condiciones minimas sin las cuales realmente seria aún mucho más difícil que sucediese ninguno de estos procesos. 
En Junio de 1997 en Cartagena de Indias, Colombia, nos reunimos especialistas de todo el mundo para repasar los trabajos que se vienen haciendo en este sentido con motivo del 20 aniversario del primer Congreso mundial de Investigación Participante. Muchas de estas investigaciones somos conscientes que han adolecido de más voluntarismo que rigor metodológico. Pero también es cierto que es mucho lo que se ha ganado en el rigor de la metodología en estos años en todo el mundo, y que tambien las perspectivas más académicas no han sabido dar cuenta mejor que los voluntarismos de los movimientos sociales, sobre todo en el plano resolutivo de los problemas de los movimientos.

Y como último argumento en favor de este replanteamiento metodológico, es la progresiva coincidencia de este tipo de paradigmas complejos y constructivistas tanto con los nuevos planteamientos de las ciencias positivas (de los que se toman muchas de sus metáforas), como con los acercamientos motivacionales de muchos de los nuevos movimientos sociales. Desde el ecologismo aparecen visiones más holísticas y eco-diversas, desde el feminismo se hacen puentes con el ecologismo, anti-militarismo, y otras confluencias sinérgicas, desde los movimientos locales y étnicos se pasa de las identidades puras a la construcción de nuevas identificaciones híbridas, los movimientos eclesiales de base, sindicales, y los más ideológicos cada vez se abren más a las otras perspectivas.

Nos parece que muchas de estas consideraciones no son las elucubraciones de un despacho citanđo autores, sino la constatación de lo que se está diciendo en los grandes Foros internacionales donde se reúnen los movimientos sociales y las organizaciones no gubernamentales. A lo largo de los años 90 este es uno de los sintomas más claros, a pesar de su aparente caos de formas e ideologías, de que se están construyendo las bases de otros valores civilizatorios en gran medida enfrentados con los que hasta ahora han venido defendiendo las conferencias gubernamentales 0 los grandes bancos y trasnacionales en sus reuniones más importantes. Aunque solo fuera por este dato valdría la pena pasar a pensar qué está pasando en el mundo con estos nuevos planteamientos. 
Juego de ejemplos de "analizadores"

de conjuntos y conductas en el tejido social

\begin{tabular}{|c|c|c|c|c|}
\hline $\begin{array}{l}\text { Conjuntos } \\
\text { de acción }\end{array}$ & $\begin{array}{c}\text { Conversas } \\
\text { (integración) }\end{array}$ & $\begin{array}{l}\text { Perversas } \\
\text { (Ruptura) }\end{array}$ & $\begin{array}{c}\text { Subversivas } \\
\text { (Ironía) }\end{array}$ & $\begin{array}{c}\text { Reversas } \\
\text { (Humor) }\end{array}$ \\
\hline Práticas & \multicolumn{2}{|c|}{ (Respuestas serias) } & \multicolumn{2}{|c|}{ (Perguntas divertidas) } \\
\hline $\begin{array}{l}\text { Desconexión } \\
\text { Auto-aislados } \\
\text { (Rip/Gafi/Siace) }\end{array}$ & $\begin{array}{l}\text { Pandila de jóvenes } \\
\text { que no cuestionan } \\
\text { nada y "pasan" } \\
\text { adaptándose }\end{array}$ & $\begin{array}{l}\text { Asociaciones que } \\
\text { critican al poder y } \\
\text { a la gente, pues } \\
\text { no les "entienden" }\end{array}$ & $\begin{array}{l}\text { Pandillas de } \\
\text { jóvenes que se } \\
\text { ríen de todo y } \\
\text { desestabilizan no } \\
\text { colaborando } \\
\end{array}$ & $\begin{array}{l}\text { Trabajadores } \\
\text { sociales } \\
\text { cumplidores, pero } \\
\text { críticos de su } \\
\text { proprio papel }\end{array}$ \\
\hline $\begin{array}{l}\text { Acción } \\
\text { Gestionalista } \\
\text { (Rip + Gafi/Siace) }\end{array}$ & $\begin{array}{l}\text { Comerciantes que } \\
\text { negocian la } \\
\text { protección policial } \\
\text { y subvenciones }\end{array}$ & $\begin{array}{l}\text { Asociaciones en } \\
\text { las comisiones de } \\
\text { participación, } \\
\text { como oposición } \\
\text { que bloquea al } \\
\text { poder }\end{array}$ & $\begin{array}{l}\text { Asociación que } \\
\text { paraliza a la gran } \\
\text { inmobobiliaria, } \\
\text { com denuncias } \\
\text { juridicas y de } \\
\text { prensa }\end{array}$ & $\begin{array}{l}\text { Ecologistas que } \\
\text { gestionan } \\
\text { denuncias sobre } \\
\text { que la administra- } \\
\text { ción no cumple } \\
\text { sus proprias leyes }\end{array}$ \\
\hline $\begin{array}{l}\text { Acción } \\
\text { Populista } \\
\text { (Rip + Siace/Gafi }\end{array}$ & $\begin{array}{l}\text { Dirigente que } \\
\text { reparte lo que da } \\
\text { el poder, desde su } \\
\text { papel jerárquico }\end{array}$ & $\begin{array}{l}\text { Acción violenta y } \\
\text { aislada que } \\
\text { interpreta los } \\
\text { deseos de la gente }\end{array}$ & $\begin{array}{l}\text { Líder feminista } \\
\text { que denuncia el } \\
\text { machismo contra } \\
\text { las amas de casa }\end{array}$ & $\begin{array}{l}\text { Cura que hace } \\
\text { cumplir la } \\
\text { legislación } \\
\text { antidroga a los } \\
\text { policias implicados }\end{array}$ \\
\hline $\begin{array}{l}\text { Acción } \\
\text { Ciudadanista } \\
\text { (Gafi + Siace/Rip) }\end{array}$ & $\begin{array}{l}\text { Movimento de } \\
\text { mujeres contra la } \\
\text { droga, y piquetes } \\
\text { ciudadanos }\end{array}$ & $\begin{array}{l}\text { Movilización de } \\
\text { indignación radical } \\
\text { por subida de pre- } \\
\text { cios o por } \\
\text { quedarse sin } \\
\text { vivienda }\end{array}$ & $\begin{array}{l}\text { Movimiento para } \\
\text { la recuperación de } \\
\text { cuarteles, para } \\
\text { dedicarlos a } \\
\text { equipamientos } \\
\text { alternativos }\end{array}$ & $\begin{array}{l}\text { Movilización para } \\
\text { dar clases en um } \\
\text { descampado o } \\
\text { plantar árboles } \\
\text { como denuncia de } \\
\text { las carencias }\end{array}$ \\
\hline
\end{tabular}

(Juéguese poniendo ejemplos concretos, de la realidad local de cada uno, en cada casillero). 
Praxis de la participación/iniciativas cuidadanas

para la integración social

\begin{tabular}{|c|c|c|c|c|}
\hline Práticas/Frases & Reglamentos & N.I.P & P. Comunitanios & IAP/PAI \\
\hline $\begin{array}{l}\text { Negociación } \\
\text { inicial }\end{array}$ & $\begin{array}{l}\text { Administración y } \\
\text { Asociaciones en } \\
\text { litigio }\end{array}$ & $\begin{array}{l}\text { Administración, } \\
\text { técnicos y } \\
\text { asociaciones en } \\
\text { litigio }\end{array}$ & $\begin{array}{l}\text { Administración } \\
\text { y/o asociaciones } \\
\text { y/o técnicos }\end{array}$ & $\begin{array}{l}\text { Administración } \\
\text { asociaciones y } \\
\text { técnicos }\end{array}$ \\
\hline Objetivos & $\begin{array}{l}\text { Complementación } \\
\text { de la democracia } \\
\text { representativa }\end{array}$ & $\begin{array}{l}\text { Consenso por } \\
\text { cada tema } \\
\text { suscitado }\end{array}$ & $\begin{array}{l}\text { Dinamización } \\
\text { comunitaria por } \\
\text { autodiagnóstico y } \\
\text { programaciones }\end{array}$ & $\begin{array}{l}\text { Dinamización } \\
\text { instituyente para } \\
\text { constitutir una } \\
\text { programación } \\
\text { movilizadora }\end{array}$ \\
\hline Grupos de trabajo & $\begin{array}{l}\text { Representantes de } \\
\text { la Administración } \\
\text { y de las } \\
\text { Asociaciones }\end{array}$ & $\begin{array}{l}\text { Selección al azar } \\
\text { de vecinos der } \\
\text { censo }\end{array}$ & $\begin{array}{l}\text { Equipo técnico en } \\
\text { contacto com los } \\
\text { dirigentes sociales }\end{array}$ & $\begin{array}{l}\text { Grupos mixtos de } \\
\text { técnicos y } \\
\text { voluntários }\end{array}$ \\
\hline Información & $\begin{array}{l}\text { Precedentes de } \\
\text { participación }\end{array}$ & $\begin{array}{l}\text { Documentación } \\
\text { técnica com } \\
\text { asesores neutrales }\end{array}$ & $\begin{array}{l}\text { Dados } \\
\text { socioeconómicos y } \\
\text { participación de } \\
\text { las asociaciones }\end{array}$ & $\begin{array}{l}\text { Datos } \\
\text { socioeconómicos, } \\
\text { mapeo del tejido } \\
\text { asociativo e informal }\end{array}$ \\
\hline Diagnóstico & $\begin{array}{l}\text { Talleres sobre } \\
\text { propuestas de } \\
\text { Regiamento }\end{array}$ & $\begin{array}{l}\text { Talleres del } \\
\text { "nucleo" com } \\
\text { expertos y } \\
\text { autoridades }\end{array}$ & $\begin{array}{l}\text { Informadores } \\
\text { privilegiados y } \\
\text { coloquios de calle }\end{array}$ & $\begin{array}{l}\text { "triangulación de } \\
\text { entrevistas y grupos } \\
\text { de discución para } \\
\text { conjuntos de } \\
\text { acción" }\end{array}$ \\
\hline Propuestas & $\begin{array}{l}\text { Projecto de } \\
\text { Regiamento y } \\
\text { Consejos }\end{array}$ & $\begin{array}{l}\text { Dictámen } \\
\text { ciudadano }\end{array}$ & $\begin{array}{l}\text { Programación de } \\
\text { distintas lineas de } \\
\text { intervención } \\
\text { sectorial }\end{array}$ & $\begin{array}{l}\text { Selección de un } \\
\text { tema generador y } \\
\text { autosustentable }\end{array}$ \\
\hline Acuerdos & $\begin{array}{l}\text { En el Pleno } \\
\text { municipal }\end{array}$ & $\begin{array}{l}\text { No vinculate para } \\
\text { la Administación }\end{array}$ & $\begin{array}{l}\text { Respaldo de } \\
\text { diferentes } \\
\text { administradores }\end{array}$ & $\begin{array}{l}\text { Comisiones pue } \\
\text { salen de una } \\
\text { Asamblea (com } \\
\text { apoyo técnico si es } \\
\text { factible) }\end{array}$ \\
\hline Ejecución & $\begin{array}{l}\text { Organograma com } \\
\text { participación de } \\
\text { Asociaciones en } \\
\text { Consejos } \\
\text { consultivos }\end{array}$ & $\begin{array}{l}\text { Acuerdo } \\
\text { consensuado de } \\
\text { las partes } \\
\text { sancionado por la } \\
\text { Administración }\end{array}$ & $\begin{array}{l}\text { Coodinación de } \\
\text { servicios abiertos } \\
\text { por la } \\
\text { Administración }\end{array}$ & $\begin{array}{l}\text { Difusión, formación, } \\
\text { tema de decisiones } \\
\text { y evaluación por las } \\
\text { asociaciones y } \\
\text { técnicos implicados }\end{array}$ \\
\hline
\end{tabular}




\section{Bibliografia}

BOURDIEU, P. El sentido práctico. Taurus: Madrid, 1991.

CHOMSKY, N. Ilusiones necesarias. Control del pensamiento en las sociedades democráticas. Madrid: Libertarias/Prodhufi, 1992.

FALS BORDA, O. El problema de como investigar la realidad para transformarla por la praxis. Bogotá: Tercer Mundo, 1989.

FREIRE, P. "Interrogantes y propuestas". Temas de Psicología Social, Buenos Aires, v. 4, n. 13, 1993.

GALTUNG, J. ¡Hay alternativas! Madrid: Tecnos, 1984.

GONZȦLEZ CASANOVA, P. La hegemonía del pueblo y la lucha centroamericana. Buenos Aires: Contrapunto, 1987.

IBAÑEZ, J. El regreso del sujeto. Madrid: Siglo XXI, 1994.

. Por una sociología de la vida cotidiana. Madrid: Siglo XXI, 1994.

LAPPASADE, G. Socioanálisis y potencial humano. Barcelona: GBDISA, 1980.

MART'́N BARBERO, J. De los medios a las mediaciones. México, G. Gili., 1987.

MARX, K. Tesis sobre Feuerbach. México: Grijalbo, 1970.

MATURANA, H. La realidad, ¿objetiva o construida? Barcelona: Anthropos, 1995.

MIRES, F. La revolución que nadie soñó o la otra posmodernidad. Caracas: Nueva Sociedad, 1996.

MORIN, E. Introducción al pensamiento complejo. Barcelona: Gedisa, 1994.

NEGRI, A. El poder constituyente. Madrid: Libertarias/Prodhufi, 1994.

NERFIN, M. Ni principe, ni mercader: ciudadano. Lima: Socialismo y Participación, 1988.

NUÑEZ, C. Educar para transformar, transformar para educar. S. José de Costa Rica: Alforja, 1989.

PRTGOGINE, MORIN, VON FOESTER et al. Nuevos paradigmas, cultura y subjetividad. Buenos Aires: Paidós, 1994.

ORTÍ, A. "La estrategia de la oferta en la sociedad neocapitalista de consumo: génesis y praxis de la investigación motivacional de la demanda". Política y Sociedad, Madrid, v. 16, 1994.

RIECHMANN, F. Buey. Redes que dan libertad. Barcelona: Paidós, 1994.

RIFKIN, J. "La sociedad civil en la era de la información”. El Viejo Topo, 97. Barcelona, 1996.

SACRISTÁN, M. Pacifismo, ecologia y política altemativa. Barcelona: Icaria, 1987.

VILLASANTE et al. Las ciudades hablan. Caracas: Nueva Sociedad, 1994.

VILLASANTE, T. R. Las democracias participativas. Madrid: HOAC, 1995. 